\title{
Performance of Direct-oversampling Correlator-type Receivers in Chaos-based DS-CDMA Systems Over Frequency Non-selective Fading Channels
}

\author{
Nguyen Xuan Quyen • Pere Barlet-Ros
}

Received: 28th Sept. 2015/ Accepted: 3rd March 2017

\begin{abstract}
In this paper, we present a study on the performance of directoversampling correlator-type receivers in chaos-based Direct-sequence code division multiple access (DS-CDMA) systems over frequency non-selective fading channels. At the input, the received signal is sampled at a sampling rate higher than the chip rate. This oversampling step is used to precisely determine the delayed-signal components from multipath fading channels, which can be combined together by a correlator for the sake of increasing the SNR at its output. The main advantage of using direct-oversampling correlator-type receivers is not only their low energy consumption due to their simple structure, but also their ability to exploit the non-selective fading characteristic of multipath channels to improve the overall system performance in scenarios with limited data speeds and low energy requirements, such as Low-rate wireless personal area networks (LR-WPAN). Mathematical models in discrete-time domain for the conventional transmitting side with multiple access operation, the generalized non-selective Rayleigh fading channel, and the proposed receiver are provided and described. A rough theoretical bit-error-rate (BER) expression is first derived by means of Gaussian approximation. We then define the main component in the expression and build its probability mass function (PMF) through numerical computation. The final BER estimation is carried out by integrating the rough expression over possible discrete values of the PFM. In order to validate our findings, PC simulation is performed and simulated performance is compared with the corresponding estimated one. Obtained results
\end{abstract}

Nguyen Xuan Quyen

School of Electronics and Telecommunications, Hanoi University of Science and Technology E-mail: quyen.nguyenxuan@hust.edu.vn

Pere Barlet-Ros

Department of Computer Architecture, UPC BarcelonaTech, Jordi Girona 1-3, 08034 Barcelona, Spain

E-mail: pbarlet@ac.upc.edu 
show that the system performance get better with the increment of the number of paths in the channel.

Keywords Chaos-based communications - Chaos-based DS-CDMA . Correlator-type receiver · Frequency non-selective fading channels · BER performance

\section{Introduction}

Pseudo-noise (PN) sequences have been widely used in direct-sequence code division multiple access (DS-CDMA) communication systems [1]. Despite their good correlation properties, the main weakness of these sequences is their limited security. For example, PN sequences can be easily reconstructed by linear regression attacks [2], due to their small linear complexity. In order to overcome this limitation, several works [3] have proposed to replace PN sequences by chaotic sequences in DS-CDMA systems. Chaotic sequences have an infinite number of states, non-periodicity and wide-band. Therefore, they are very difficult to predict, reproduce, and intercept [3]-[5]. Interestingly, the generation of an infinitive number of uncorrelated chaotic sequences for multi-user operation can be simply carried out by slightly changing the initial values [6]-[8]. Instead of using continuous chaotic sequences as in $[4,6]$, the studies in $[9,10]$ proposed the adoption of complex multilevel spreading sequences generated by repeating truncated and quantized chaotic time series for spreading the data symbols. Obtained results show that DS-CDMA systems using periodic quantized sequences also have larger capacity and better performance than those using Gold and $m$-sequences in a multiple-access environment.

Over the last two decades, multiple works have been devoted to the design and analysis of chaos-based DS-CDMA systems. These studies can be divided into two main groups: (1) those that addressed the design [11], optimization [12], and synchronization of communication systems for chaotic spreading sequences [13]-[16]; and (2) those that provided a theoretical and numerical analysis of the BER performance for multiple-access operation under different transmission channels [17]-[27]. Most of these previous works used correlator-type receivers to study the BER performance over noisy channels $[4$, 6], [17]-[23], because of their simple architecture. In contrast, the performance of chaos-based DS-CDMA systems over multipath fading channels has only been investigated by means of more complicated RAKE receivers, which can combine multipath components to enhance the signal-to-noise ratio [24]-[27].

In this paper, we address the design of a secure physical layer for wirelessbased multiple-access applications with limited data speed and low energy consumption in small transmission areas [31], such as wireless sensor networks (WSNs) or low-rate wireless personal area networks (LR-WPANs) [32,33]. Due to the short transmission distance and limited data rates in these scenarios, the root-mean-square (RMS) delay spread is less than the symbol duration, hence the wireless transmission environment can be considered as a frequency non-selective fading channel (or flat fading channel) [34]. We argue that, under 
these conditions, direct-oversampling correlator-type receivers could be used as an alternative to the more complex RAKE receivers proposed in previous works [24]-[27]. The utilization of direct-oversampling correlator-type receivers in these scenarios has two main advantages: (1) they have low energy consumption due to their simple architecture, and (2) they can better exploit the flat fading characteristic of the channels in order to improve the system performance.

To the best of our knowledge, this is the first work to study the BER performance of chaos-based DS-CDMA systems over multipath channels having a frequency non-selective fading characteristic, when direct-oversampling correlator-type receivers are used. In the receiver, we propose to sample the incoming signal at a rate higher than the chip rate, before entering the correlator. This oversampling step aims to specifically determine the delayed-signal components from fading multipath channels, which can be combined together by the correlator in order to increase the signal-to-noise ratio at its output. The application of an oversampling step at the receiver side has been shown to improve the communication features in [28]-[30].

The most relevant methods for the theoretical calculation of the BER performance for chaos-based DS-CDMA systems over noisy and multipath fading channels are presented in Section II. We find that BER expressions of high accuracy methods are very complicated and usually written in the form of multiple integral, thus requiring very complex calculations. In order to overcome this drawback, in this paper, we estimate the BER performance in two steps: theoretical derivation and discrete integral. Firstly, we derive a rough BER expression in closed form through Gaussian approximation by assuming that the output signal of the integrator is a random variable being the sum of statistical independent components. Secondly, we take into account the variation of the fading coefficients by computing the probability mass function (PMF) for the ratio of variable bit energy to noise power spectral density in the rough expression. Finally, we calculate the estimated BERs by numerically integrating the rough expression over all the discrete values of the PMF.

The main contributions of this paper can be summarized as follows:

- We propose and investigate the novel idea of applying a direct-sampling technique to conventional correlator-type receivers for enhancing the performance of chaos-based DS-CDMA communication systems over frequency non-selective fading channels. The operation of the receiver is described by means of building and analyzing the mathematical model of the whole system in discrete-time domain. The performance estimation is carried out by means of both theoretical derivation and numerical computation. We verify the proposed model and estimation through simulation.

- We use a generalized flat Rayleigh fading channel for the first time in the evaluation of the receiver performance. The discrete-time model and the typical parameters of the channel are presented. The impact of the channel on the system performance is determined in terms of the BER value against 
these typical parameters. Our results show that the more the number of paths in the channel increases, the better the system performs.

The rest of this paper is organized as follows. A review of the related work is provided in Section II. In Section III, we build and describe the mathematical modeling of the system. The methodology to estimate the BER performance is presented in Section IV. The simulation and estimation results are shown and discussed in Section V. Finally, the conclusions are given in Section VI.

\section{Related Work}

With respect to noisy channels, the performance of a chaos-based DS-CDMA system was investigated for the first time in $[4,6]$ only by means of numerical simulations. Many subsequent studies focused on estimating the BER performance theoretically for this system. The simplest estimation is presented in [17] under the assumption that the bit energy is constant. Another approach is proposed in $[18,19]$, where the decision parameter at the output of the correlator is considered as a Gaussian variable. These estimation methods are only valid when the spreading factors (SFs) are very high, while for low and moderated SFs they become seriously imprecise. The cause of this inaccuracy is the non-periodic nature of chaotic sequences, as the bit energy of the transmitted signal varies from one bit to another. In order to take into account the dynamic behavior of the chaotic sequence, the approach in $[20,21]$ integrates the BER expression for a given spreading factor over all possible sequences. This method provides an accurate estimation even for small SFs, but it requires very complex calculations. A method developed later in [22,23] relies on computing the probability density function (PDF) of the bit energy variable and then integrating the BER expression over all possible PDF values. Although this method has high accuracy, its BER expression is in the form of a multiple integral. It is important to note that in all estimation methods described above, correlator-type receivers are exploited and the reproduced chaotic sequences are assumed to be in a synchronous state.

Most of the recent studies on chaos-based DS-CDMA systems have focused on investigating the BER performance of the system over fading, multipath, and multipath fading channels [24]-[27]. In order to overcome the severe consequences of multiple channels, Rake receivers were used under the assumption that both the sequence synchronization and the channel estimation are perfect. A chaos-based DS-CDMA system under a multipath fading channel was analyzed and measured in $[24,25]$. The symbols were first separated from inter-symbol and inter-user interference parts. The BER expression was then derived in a quite complicated form by using Gaussian approximation. Based on the approach of computing the bit energy PDF as in [23], accurate estimations of the BER performance of multi-user systems under multipath and fading channels were presented in [26] and [27], respectively. However, when the number of users and paths increases, the theoretical determination of the $\mathrm{PDF}$ and the calculation of the BER expression with multiple integral become 
very complicated.

Several studies on the design of communication systems at the physical layer for WSNs and LR-WPANs have been carried out recently, mainly based on the IEEE Standard 802.15.4 [32,33]. The studies related to the design of a modulator/demodulator and a complete transceiver are presented in [35] and [36], respectively. The work in [37] presents an investigation using cooperative orthogonal frequency division multiplexing (OFDM) for semi distributed detection in WSNs. A low power cooperative multiple-input multiple-output (MIMO) scheme with low density parity check channel codes is proposed in [38]. The design of the physical layer for WSNs based on conventional DSCDMA systems using the chip interleaving technique is investigated in [31]. The latest study on a chaos-based DS-CDMA systems has been published in [39]. The multipath fading model used there is a wide-band channel where both of the fading coefficients and multipath delays vary according to random distributions. The BER expression is given in the closed form. However, simulation results are only shown for one special case, with significant differences with respect to the theoretical analysis.

\section{Mathematical Modeling of The System}

This section presents the mathematical modeling of the chaos-based DS-CDMA system under study. Fig. 1 shows the block diagram of the system. The schemes for the transmitting side with $K$ users and the generalized frequency nonselective channel with $L$ delayed paths are displayed in Fig. 1(a), while the scheme for the proposed direct-oversampling correlator-type receiver is shown in Fig. 1(b).

\subsection{Model of Transmitting Side with Multiple-access Operation}

The input bit streams of the $K$ users are spread by $K$ uncorrelated chaotic sequences produced by $K$ chaotic generators, which use the same chaotic map with different initial conditions. Here, $b_{l}^{(i)}$ and $x_{k, l}^{(i)}$ respectively denote the $l^{t h}$ bit of the $i^{\text {th }}$ user and the $k^{\text {th }}$ chip in the $l^{\text {th }}$ bit of the $i^{\text {th }}$ user. Both $b_{l}^{(i)}$ and $x_{k, l}^{(i)}$ are equal to either +1 or -1 . The ratio $\beta=T_{b} / T_{c}$ is called the spreading factor, i.e., the number of chips per bit, where $T_{b}$ and $T_{c}$ are the bit and chip duration, respectively. The output signal of the $i^{t h}$ user in the $k^{t h}$ chip duration is given by

$$
c_{k, l}^{(i)}=b_{l}^{(i)} x_{k, l}^{(i)} \text {. }
$$

The signal transmitted on the channel is the sum of all output signals from the $K$ transmitters, which can be expressed as follows:

$$
e_{k, l}=\sum_{i=1}^{K} c_{k, l}^{(i)}=\sum_{i=1}^{K} b_{l}^{(i)} x_{k, l}^{(i)}
$$




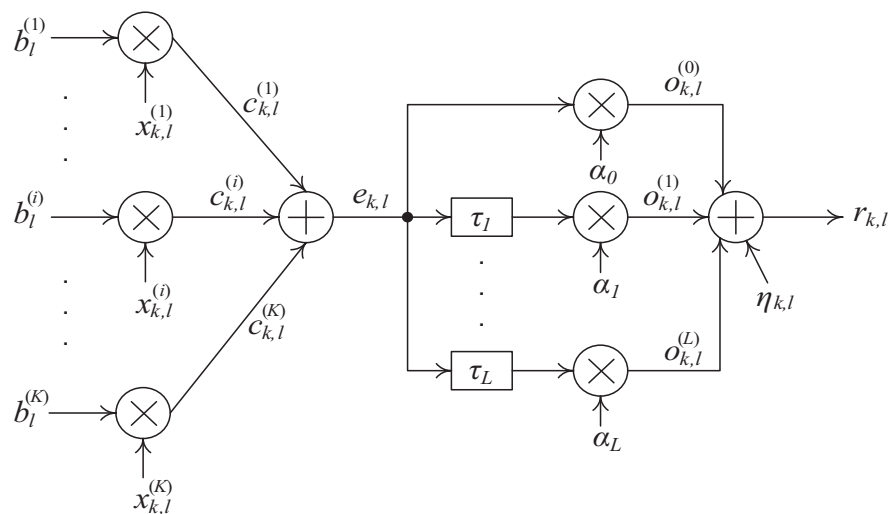

(a)

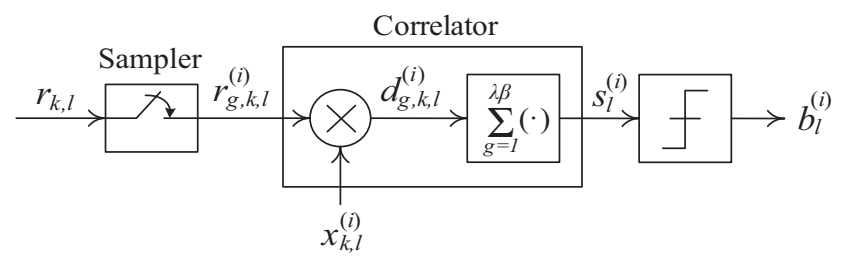

(b)

Fig. 1 Block diagram of the chaos-based DS-CDMA communication system under study: (a) The transmitting side with multiple-access operation and the generalized frequency nonselective fading channel, (b) The proposed direct-oversampling correlator-type receiver

\subsection{Model of Generalized Frequency Non-selective Fading Channel}

The transmitted signal can reach the receiver from various paths with various delays and attenuation. The path having the shortest transmission period is considered as the primary path with delay being zero, while the rest are the secondary paths. The channel model investigated here is assumed to have $L$ secondary paths with impulse response being

$$
h(n)=\sum_{j=0}^{L} \alpha_{j} \delta\left(n-\tau_{j}\right),
$$

where $\alpha_{j}$ is the fading coefficient of the $j^{\text {th }}$ path, which varies randomly according to a Rayleigh distribution [40] given by

$$
f\left(\alpha_{j}\right)=\frac{\alpha_{j}}{\sigma_{j}^{2}} e^{-\alpha_{j}^{2} /\left(2 \sigma_{j}^{2}\right)},
$$

with $\sigma_{j}$ being the scale parameter of the distribution. The mean value of each fading coefficient is determined by $\bar{\alpha}_{j}=E\left[\alpha_{j}\right]=\sigma_{j} \sqrt{\pi / 2} ; \tau_{j}$ is the delay of the $j^{t h}$ path and $\delta(t)$ is the Dirac impulse. Because of the non-selective fading characteristic, the channel delays are assumed to be less or equal to the chip 


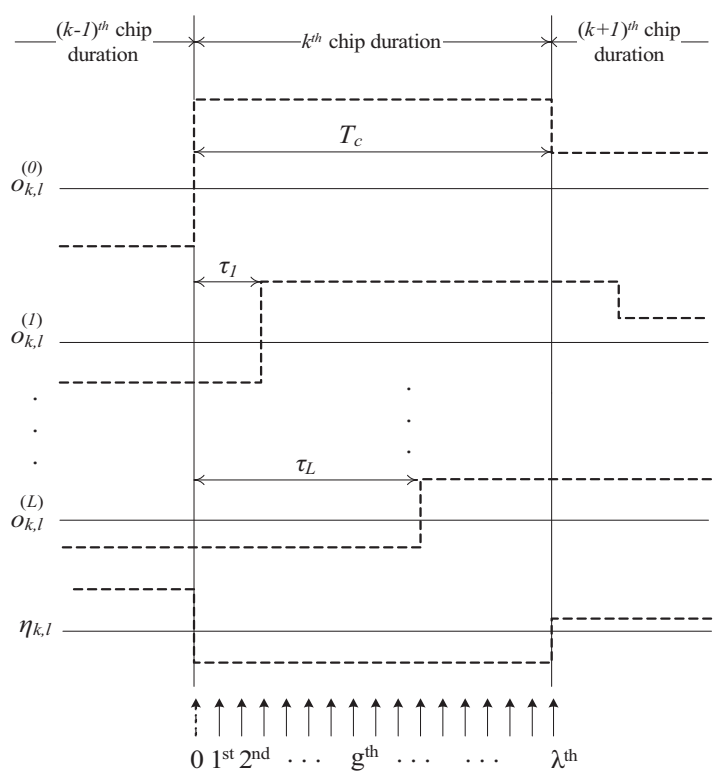

Fig. 2 Illustration of the signal components at the output of the channel and their sampling process in the receiver

duration, i.e., $\tau_{0}=0 \leq \tau_{1} \ldots \leq \tau_{j} \ldots \leq \tau_{L} \leq T_{c}$. The output signal of the channel can be written as a sum of three components as follows:

$$
r_{k, l}=r_{k, l}^{S}+r_{k, l}^{I}+\eta_{k, l}
$$

here, $r_{k, l}^{S}$ is considered as the beneficial component, which is composed of the signal from the primary path and its delayed versions from the secondary paths; $r_{k, l}^{I}$ is the interference component, which is the delayed version from the secondary paths of the previous chip duration; and $\eta_{k, l}$ is additive white Gaussian noise (AWGN). The components $r_{k, l}^{S}$ and $r_{k, l}^{I}$ are respectively determined by

$$
r_{k, l}^{S}=\alpha_{0} \sum_{i=1}^{K} b_{l}^{(i)} x_{k, l}^{(i)}+\alpha_{1} \sum_{i=1}^{K} b_{l, \tau_{1}}^{(i)} x_{k, l, \tau_{1}}^{(i)}+\ldots+\alpha_{L} \sum_{i=1}^{K} b_{l, \tau_{L}}^{(i)} x_{k, l, \tau_{L}}^{(i)}
$$

and

$$
r_{k, l}^{I}=\alpha_{1} \sum_{i=1}^{K} b_{l, \tau_{1}}^{(i)} x_{k-1, l, \tau_{1}}^{(i)}+\ldots+\alpha_{L} \sum_{i=1}^{K} b_{l, \tau_{L}}^{(i)} x_{k-1, l, \tau_{L}}^{(i)}
$$

where $b_{l, \tau_{j}}^{(i)}$ and $x_{k, l, \tau_{j}}^{(i)}$ are $b_{l}^{(i)}$ and $x_{k, l}^{(i)}$ after being delayed by a period $\tau_{j}$, respectively. 
3.3 Model of Proposed Direct-oversampling Correlator-type Receiver

In the $i^{t h}$ receiver, the incoming signal $r_{k, l}$ is first oversampled with a rate higher than the chip rate. Here, we assume that there are $\lambda$ samples per chip. The objective of this sampling step is to determine more exactly the values of the three components above in a bit duration $T_{b}$. The higher the value of $\lambda$, the higher the achieved accuracy. Fig. 2 illustrates components of the received signal $r_{k, l}$ and the oversampling process within the duration of $k^{\text {th }}$ chip in the second interval of $l^{\text {th }}$ bit. The despread-spectrum process is then carried out by multiplying the sampling signal $r_{g, k, l}$ with the local chaotic sequence $x_{k, l}^{(i)}$, which is reproduced in the same way as in the transmitter and synchronized with the incoming signal by means of the synchronization methods proposed in [13]-[16]. Here, the integrate-and-dump block operates similarly as a sum block. The consecutive samples at the input are added together in each bit duration to produce, at the output of the correlator, the signal $s_{l}^{(i)}$ as expressed by

$$
s_{l}^{(i)}=\sum_{g=1}^{\lambda \beta} d_{g, k, l}^{(i)}=X+Y+Z
$$

where the three components $X, Y, Z$ respectively are

$$
\begin{gathered}
X=\left(\sum_{j=0}^{L} \lambda \alpha_{j}\right)\left(\sum_{k=1}^{\beta} b_{l}^{(i)} x_{k, l}^{(i)}+\sum_{k=1}^{\beta} \sum_{m=1, m \neq i}^{K} b_{l}^{(m)} x_{k, l}^{(m)} x_{k, l}^{(i)}\right) \\
Y=\left(\sum_{j=1}^{L} \tau_{j} \alpha_{j}\right)\left(-\sum_{k=1}^{\beta} b_{l}^{(i)} x_{k, l}^{(i)}-\sum_{k=1}^{\beta} \sum_{m=1, m \neq i}^{K} b_{l}^{(m)} x_{k, l}^{(m)} x_{k, l}^{(i)}+\sum_{k=1}^{\beta} \sum_{m=1, m \neq i}^{K} b_{l}^{(m)} x_{k-1, l}^{(m)} x_{k, l}^{(i)}\right),
\end{gathered}
$$

and

$$
Z=\lambda \sum_{k=1}^{\beta} \eta_{k, l} x_{k, l}^{(i)}
$$

(The detailed development of Eq. (8) is presented in Appendix A)

In Eqs. (9)-(11), we can find that $Z$ is the noise component created by the AWGN. The sum of $(X+Y)$ can be rewritten in another form as follows:

$$
X+Y=S+I,
$$

where $S$ is the component of the beneficial signal determined by

$$
S=\left(\sum_{j=0}^{L}\left(\lambda-\tau_{j}\right) \alpha_{j}\right) \sum_{k=1}^{\beta} b_{l}^{(i)} x_{k, l}^{(i)^{2}},
$$


and $I$ is the interference component between different users determined by

$$
\begin{aligned}
I & =\left(\sum_{j=0}^{L}\left(\lambda-\tau_{j}\right) \alpha_{j}\right) \sum_{k=1}^{\beta} \sum_{m=1, m \neq i}^{K} b_{l}^{(m)} x_{k, l}^{(m)} x_{k, l}^{(i)} \\
& +\left(\sum_{j=1}^{L} \tau_{j} \alpha_{j}\right) \sum_{k=1}^{\beta} \sum_{m=1, m \neq i}^{K} b_{l}^{(m)} x_{k-1, l}^{(m)} x_{k, l}^{(i)},
\end{aligned}
$$

Based on Eqs. (11), (13), (14), it is easy to find that the values of the components $S, I$, and $Z$ increase when the number of delay paths $L$ or the number of samples per chip $\lambda$ increase. However, owing to the low cross-correlation between the different chaotic sequences, and between the chaotic sequences and AWGN, the increasing amount of the components $I$ and $Z$ is much less than that of the component $S$. Therefore, the SNR of the output signal $s_{l}^{(i)}$ increases. The increment of the SNR leads to the improvement of the system performance.

The binary value of the $l^{\text {th }}$ bit is finally recovered by the decision circuit using a sign function as follows:

$$
b_{l}^{(i)}=\left\{\begin{aligned}
1 & s_{l}^{(i)} \geq 0 \\
-1 & s_{l}^{(i)}<0 .
\end{aligned}\right.
$$

\section{Estimation of the BER Performance}

In this section, the two steps proposed to estimate the BER performance of the studied system are presented. With the assumptions that the fading coefficients and the bit energy are fixed, a rough BER expression is first derived by means of Gaussian approximation. We then define the main component in the expression and build its PMF by numerical computation. The final BER estimation is carried out by integrating the rough expression over possible discrete values of the PFM.

\subsection{Theoretical Derivation}

Assuming that each bit, either " +1 " or " -1 ", appears at the output of the data source with a probability of $1 / 2$, we first determine the mathematical statistics of the components, i.e., $X, Y$ and $Z$, in the case that a +1 bit is transmitted. The mean values are determined as follows (see Appendix B):

$$
\begin{gathered}
E\left[X \mid b_{l}^{(i)}=+1, \alpha_{j}\right]=\left(\sum_{j=0}^{L} \lambda \alpha_{j}\right) \beta E_{c}, \\
E\left[Y \mid b_{l}^{(i)}=+1, \alpha_{j}\right]=-\left(\sum_{j=1}^{L} \tau_{j} \alpha_{j}\right) \beta E_{c},
\end{gathered}
$$


and

$$
E\left[Z \mid b_{l}^{(i)}=+1, \alpha_{j}\right]=\lambda E\left[\sum_{k=1}^{\beta} \eta_{k, l} x_{k, l}^{(i)}\right]=0 .
$$

The mean squared values are derived as (see Appendix C)

$$
\begin{gathered}
E\left[X^{2} \mid b_{l}^{(i)}=+1, \alpha_{j}\right]=\left(\sum_{j=0}^{L} \lambda \alpha_{j}\right)^{2}\left(\beta E_{c 4}+\beta(\beta+K-2) E_{c}^{2}\right), \\
E\left[Y^{2} \mid b_{l}^{(i)}=+1, \alpha_{j}\right]=\left(\sum_{j=1}^{L} \tau_{j} \alpha_{j}\right)^{2}\left(\beta E_{c 4}+\beta(\beta+2 K-2) E_{c}^{2}\right), \\
E\left[Z^{2} \mid b_{l}^{(i)}=+1\right]=\lambda^{2} E_{b} \frac{N_{0}}{2} .
\end{gathered}
$$

In the above equations, $E_{c}=E\left[x_{k, l}^{(i)^{2}}\right], E_{c 4}=E\left[x_{k, l}^{(i)^{4}}\right], E_{b}=\beta E_{c}$ and $N_{0}=$ $2 E\left[\eta_{k, l}^{2}\right]$ are the bit energy and noise power spectral density, respectively.

Using the mean and mean squared values obtained above, the variances of $X$, $Y, Z$ are calculated by

$$
\begin{aligned}
\operatorname{Var}\left[X \mid b_{l}^{(i)}=+1, \alpha_{j}\right] & =E\left[X^{2} \mid b_{l}^{(i)}=+1\right]-E^{2}\left[X \mid b_{l}^{(i)}=+1\right] \\
& =\left(\sum_{j=0}^{L} \lambda \alpha_{j}\right)^{2}\left(\beta E_{c 4}+\beta(K-2) E_{c}^{2}\right), \\
\operatorname{Var}\left[Y \mid b_{l}^{(i)}=+1, \alpha_{j}\right] & =E\left[Y^{2} \mid b_{l}^{(i)}=+1\right]-E^{2}\left[Y \mid b_{l}^{(i)}=+1\right] \\
& =\left(\sum_{j=1}^{L} \tau_{j} \alpha_{j}\right)^{2}\left(\beta E_{c 4}+\beta(2 K-2) E_{c}^{2}\right),
\end{aligned}
$$

and

$$
\operatorname{Var}\left[Z \mid b_{l}^{(i)}=+1\right]=E\left[Z^{2} \mid b_{l}^{(i)}=+1\right]-E^{2}\left[Z \mid b_{l}^{(i)}=+1\right]=\lambda^{2} E_{b} \frac{N_{0}}{2} .
$$

Due to the physical characteristics of the communication system, in Eqs. (9)(11), the binary data $b_{l}^{(i)}=\{ \pm 1\}$, channel delays $\tau_{j}$, number of paths $L$, number of users $K$, number of samples $\lambda$, and spreading factor $\beta$ are constants, while the parameters, i.e., chaotic sequence $x_{k, l}^{(i)}$, fading coefficient $\alpha_{j}$, and Gaussian noise $\eta_{k, l}$, are assumed to be independent random variables. Under this condition, the components, i.e., $\left(\sum_{j=0}^{L} \lambda \alpha_{j}\right)$ and $\left(\sum_{k=1}^{\beta} b_{l}^{(i)} x_{k, l}^{(i)}{ }^{2}+\right.$ $\left.\sum_{k=1}^{\beta} \sum_{m=1, m \neq i}^{K} b_{l}^{(m)} x_{k, l}^{(m)} x_{k, l}^{(i)}\right)$ of the variable $X,\left(\sum_{j=1}^{L} \tau_{j} \alpha_{j}\right)$ and $\left(-\sum_{k=1}^{\beta} b_{l}^{(i)} x_{k, l}^{(i)^{2}}-\right.$ 
$\left.\sum_{k=1}^{\beta} \sum_{m=1, m \neq i}^{K} b_{l}^{(m)} x_{k, l}^{(m)} x_{k, l}^{(i)}+\sum_{k=1}^{\beta} \sum_{m=1, m \neq i}^{K} b_{l}^{(m)} x_{k-1, l}^{(m)} x_{k, l}^{(i)}\right)$ of the variable $Y$, and $Z=\left(\lambda \sum_{k=1}^{\beta} \eta_{k, l} x_{k, l}^{(i)}\right)$ of the variable $Z$, are considered as mutually independent random variables. Therefore, the variables, i.e., $X, Y$, and $Z$ are also statistically independent. The mean value and variance of the signal $s_{l}^{(i)}$ are given as

$$
E\left[s_{l}^{(i)} \mid b_{l}^{(i)}=+1, \alpha_{j}\right]=E\left[X \mid b_{l}^{(i)}=+1, \alpha_{j}\right]+E\left[Y \mid b_{l}^{(i)}=+1, \alpha_{j}\right]+0,
$$

and

$$
\operatorname{Var}\left[s_{l}^{(i)} \mid b_{l}^{(i)}=+1, \alpha_{j}\right]=\operatorname{Var}\left[X \mid b_{l}^{(i)}=+1, \alpha_{j}\right]+\operatorname{Var}\left[Y \mid b_{l}^{(i)}=+1, \alpha_{j}\right]+\operatorname{Var}\left[Z \mid b_{l}^{(i)}=+1\right] .
$$

Analogously, the case of transmitting a " $-1 "$ bit is calculated in the same way:

$$
\begin{gathered}
E\left[s_{l}^{(i)} \mid b_{l}^{(i)}=-1, \alpha_{j}\right]=-E\left[s_{l}^{(i)} \mid b_{l}^{(i)}=+1, \alpha_{j}\right], \\
\operatorname{Var}\left[s_{l}^{(i)} \mid b_{l}^{(i)}=-1, \alpha_{j}\right]=\operatorname{Var}\left[s_{l}^{(i)} \mid b_{l}^{(i)}=+1, \alpha_{j}\right] .
\end{gathered}
$$

According to the central limit theorem (CLT) [41], if $S$ is the sum of mutually independent random variables, then the distribution function of $S$ is wellapproximated by a certain type of continuous function known as a Gaussian (or Normal) density function. Owing to the output $s_{l}^{(i)}=X+Y+Z$ with $X, Y, Z$ being mutually independent random variables, and according to the CLT, the random variable $s_{l}^{(i)}$ approximately has a Gaussian distribution. On the basic of Eqs. (15)-(27) and the results in [18]-[23], [29], the rough BER expression can be derived as follows (see Appendix D):

$$
\begin{aligned}
B E R_{R} & =\frac{1}{2} \operatorname{Prob}\left(s_{l}^{(i)} \leq 0 \mid b_{l}^{(i)}=+1, \alpha_{j}\right)+\frac{1}{2} \operatorname{Prob}\left(s_{l}^{(i)}>0 \mid b_{l}^{(i)}=-1, \alpha_{j}\right) \\
& =Q\left(\frac{\operatorname{Var}\left[s_{l}^{(i)} \mid b_{l}^{(i)}=+1, \alpha_{j}\right]}{E^{2}\left[s_{l}^{(i)} \mid b_{l}^{(i)}=+1, \alpha_{j}\right]}\right)^{-\frac{1}{2}}=Q\left(A+B+\frac{1}{2 C}\right)^{-\frac{1}{2}},
\end{aligned}
$$

where the function $Q(\cdot)$ is defined by $Q(\epsilon)=\frac{1}{\sqrt{2 \pi}} \int_{\epsilon}^{\infty} \exp \left(y^{2} / 2\right) d y$ and the components $A, B, C$ are determined as

$$
\begin{aligned}
A & =\left(\frac{\frac{E_{c 4}}{E_{c}^{2}}+K-2}{\beta}\right)\left(1-\sum_{j=1}^{L} \frac{\tau_{j}}{\lambda} \alpha_{j} / \sum_{j=0}^{L} \alpha_{j}\right)^{-2}, \\
B & =\left(\frac{\frac{E_{c 4}}{E_{c}^{2}}+2 K-2}{\beta}\right)\left(\sum_{j=0}^{L} \alpha_{j} / \sum_{j=1}^{L} \frac{\tau_{j}}{\lambda} \alpha_{j}-1\right)^{-2},
\end{aligned}
$$


and

$$
C=\left(\sum_{j=0}^{L} \alpha_{j}-\sum_{j=1}^{L} \frac{\tau_{j}}{\lambda} \alpha_{j}\right)^{2} \frac{E_{b}}{N_{0}}
$$

This rough expression is valid only if the fading coefficients and the bit energy are constant. In fact, in our system, the fading coefficients vary randomly with a defined distribution and the bit energy varies according to a given chaotic behavior. The previous studies in [24]-[27] show that, in a fading multipath channel, the variation of bit energy mainly depends upon the fading. Moreover, they show that for a large spreading factor, the effect of chaotic behavior on bit energy is not significant. Therefore, in our approach, we assume that the variation of the components within the bracket in Eq. (29), i.e., $A, B$, $C$, is affected mainly by the fading. Here, the variable $C$ is considered as the main component, which is the ratio of variable bit energy, i.e., $E_{v b}=$ $\left(\sum_{j=0}^{L} \alpha_{j}-\sum_{j=1}^{L} \frac{\tau_{j}}{\lambda} \alpha_{j}\right)^{2} E_{b}$, to noise power spectral density $N_{0}$. The variable bit energy $E_{v b}$ fully depends on the fixed bit energy $E_{b}$ and channel parameters, i.e., $L, \alpha_{j}, \tau_{j}$. For the sake of simplicity, we consider $A, B$ equal to their average values $\bar{A}, \bar{B}$ respectively, which are obtained by replacing $\alpha_{j}$ in $A$ and $B$ by its mean value, i.e., $\bar{\alpha}_{j}=E\left[\alpha_{j}\right]$. The rough expression is first approximated by

$$
B E R_{R} \approx Q\left(\bar{A}+\bar{B}+\frac{1}{2 C}\right)^{-\frac{1}{2}}
$$

after that, the conditional BER expression reflecting the variation of $C$ is derived as follows:

$$
B E R(C) \approx \int_{0}^{\infty} Q\left(\bar{A}+\bar{B}+\frac{1}{2 C}\right)^{-\frac{1}{2}} f(C) d C
$$

with $f(C)$ being the probability density function (PDF) of $C$.

An application Example: Let's determine the BER value of the system in a particular case, where the studied channel is an one-path Rayleigh fading channel. For this case, the number of secondary channels $L=0$ and the fading coefficient $\alpha_{0}$ varies according to a Rayleigh distribution given by $f\left(\alpha_{0}\right)=$ $\frac{\alpha_{0}}{\sigma_{0}^{2}} e^{-\alpha_{0}^{2} /\left(2 \sigma_{0}^{2}\right)}$, with $\sigma_{0}$ being the scale parameter of the distribution [37]. Based on Eqs. (30)-(32), we have $\bar{A}=\left(\frac{E_{c 4}}{E_{c}^{2}}+K-2\right) / \beta, B=0$ and $C=\alpha_{0}^{2} \frac{E_{b}}{N_{0}}$. The PDF of $C$ is obtained by $f(C)=\frac{1}{E[C]} e^{-\frac{C}{E[C]}}$ with $E[C]=E\left[\alpha_{0}^{2}\right] \frac{E_{b}}{N_{0}}=$ $2 \sigma_{0}^{2} \frac{E_{b}}{N_{0}}$ [37]. The BER value is then determined by means of the conditional 
BER expression as follows:

$$
\begin{aligned}
B E R(C) & \approx \int_{0}^{\infty} Q\left(\bar{A}+\frac{1}{2 C}\right)^{-\frac{1}{2}} \frac{1}{E[C]} e^{-\frac{C}{E[C]} d C} \\
& =\int_{0}^{\infty} Q\left(\frac{\frac{E_{c 4}}{E_{c}^{2}}+K-2}{\beta}+\frac{1}{2 \alpha_{0}^{2} \frac{E_{b}}{N_{0}}}\right)^{-\frac{1}{2}} \frac{e^{-\frac{\alpha_{0}^{2}}{2 \sigma_{0}^{2}}}}{\sigma_{0}^{2}} d \alpha_{0} .
\end{aligned}
$$

In fact, our studied channel is the generalized case of a frequency nonselective fading channel with multiple primary paths, i.e., $L \geq 1$. Therefore, as the number of secondary paths $L$ increases, the theoretical determination of the $f(C)$ becomes more complicated, even impossible. For this reason, another simpler approach based on numerical computation to determine the PMF of $C$, which can be considered as the discrete PDF of $C$, is presented in the next subsection, where the BER performance is estimated by means of discrete numerical integral.

\subsection{Discrete Integral}

Firstly, the use of numerical computation to build the PMF of $C$ is described. The total number of samples $M$ generated for the statistic computation, and the number of points $N$ for plotting the PMF, are chosen with proper values. Since each secondary path has a defined distribution function $f\left(\alpha_{j}\right)$ and a given mean value $\bar{\alpha}_{j}=E\left[\alpha_{j}\right]$, we first generate $M$ discrete samples for each corresponding coefficient, by using a numerically programmable tool (e.g., Matlab), which are expressed by a $M$-element vector,

$$
\vec{\alpha}^{(j)}=\left[\begin{array}{llll}
\alpha_{1}^{(j)} & \alpha_{2}^{(j)} & \ldots & \alpha_{M}^{(j)}
\end{array}\right]
$$

and then a vector $\vec{D}$ is created by

$$
\vec{D}=\sum_{j=0}^{L} \vec{\alpha}^{(j)}-\sum_{j=1}^{L} \frac{\tau_{j}}{\lambda} \vec{\alpha}^{(j)}
$$

Based on Eq. (31), the vector for the $M$ discrete samples of the variable $C$ is produced by

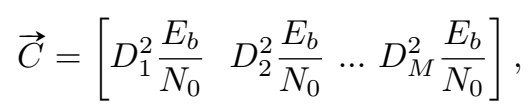

where $D_{p}$ denotes the $p^{t h}$ element of the vector $\vec{D}$.

In theory, the variation range of variable $C$ is $(0, \infty)$. However, in our numerical approach, the variation is assumed to be in a limited range of $\left(0, C_{\max }\right]$, where $C_{\max }$ is chosen with a large enough value to guarantee the estimation accuracy. The range of $\left(0, C_{\max }\right]$ is divided into $N$ equal intervals, i.e., $\left(0, \frac{C_{\max }}{N}\right]$, 


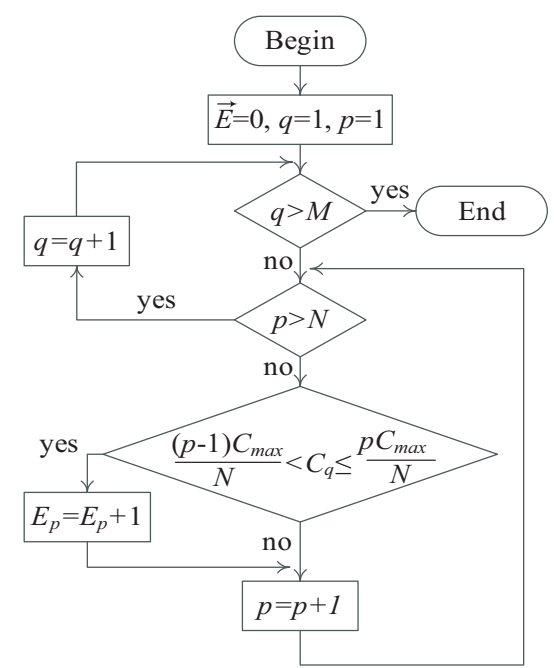

Fig. 3 Algorithm for the calculation of $\mathrm{N}$ elements in the vector $\mathbf{E}$

$\left(\frac{C_{\max }}{N}, \frac{2 C_{\max }}{N}\right], \ldots,\left(\frac{(p-1) C_{\max }}{N}, \frac{p C_{\max }}{N}\right], \ldots,\left(\frac{(N-1) C_{\max }}{N}, \frac{N C_{\max }}{N}\right]$.

The number of samples, denoted by element $E_{p}$ in vector $\vec{E}$, from the $M$ samples in vector $\vec{C}$ having a value falling into each corresponding interval $\left(\frac{(p-1) C_{\max }}{N}, \frac{p C_{\max }}{N}\right]$ are calculated by the algorithm presented in Fig. 3. From the vector $\vec{E}$ obtained, the element $P_{p}$ of vector $\vec{P}$, which denotes the probability to the variable $C$ having a value in the interval $\left(\frac{(p-1) C_{\max }}{N}, \frac{p C_{\max }}{N}\right]$, is computed by

$$
\vec{P}=\frac{\vec{E}}{M}
$$

The graph for the PMF of $C$ is created by plotting $P_{p}$ versus the centered value of each interval, i.e., $C_{p}^{*}=\frac{(p-1 / 2) C_{\max }}{N}$, for $p$ from 1 to $N$. The BER estimation is finally carried out by discretely integrating the approximated BER expression in Eq. (33) over $N$ points of the PMF as follows:

$$
B E R \approx \sum_{p=1}^{N} Q\left(\bar{A}+\bar{B}+\frac{1}{2 C_{p}^{*}}\right)^{-\frac{1}{2}} P_{p}
$$

It is noted that the higher the chosen values of $M$ and $N$, the more accurate the BER estimation, however the larger the computation time is and vice versa. Therefore, the choice of the values $M$ and $N$ has to guarantee an acceptable accuracy with an allowable period of computation time. 


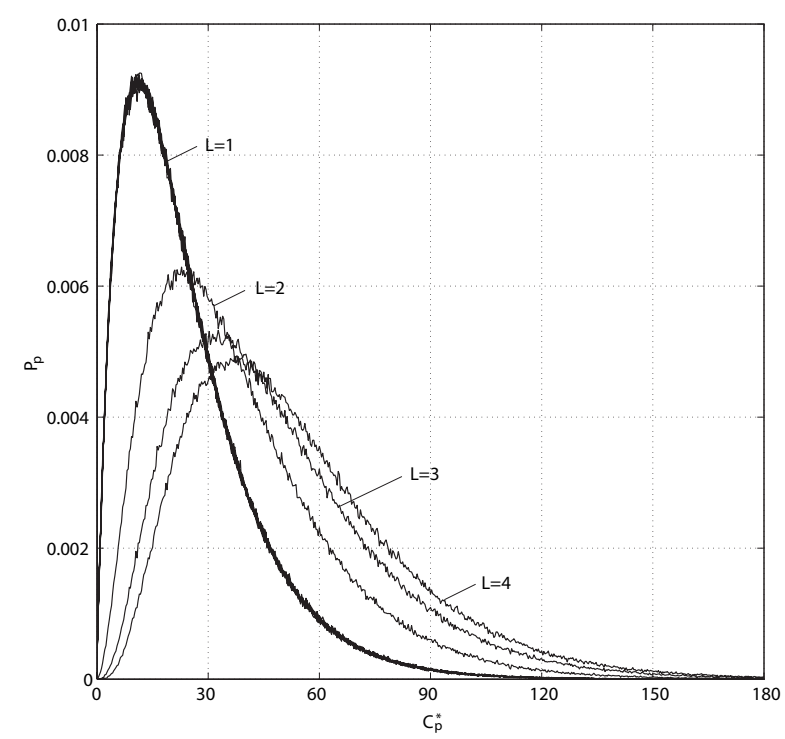

Fig. 4 Probability mass function (PMF) of the variable $C$ in the case of $\beta=64, \lambda=$ $30, E_{b} / N_{0}=10 d B$ with the increment of the number of secondary paths $L$.

\section{Simulation Results}

In this section, numerical results obtained by computer simulations with different specific parameters are presented to validate the estimation results derived in Eq. (40). The simulation parameters are given as follows:

- The chaotic map used for generating the chaotic sequences is the Chebyshev polynomial function of order 2, i.e., $x_{k}=f\left(x_{k-1}\right)=2 x_{k-1}^{2}-1$ with $E_{c}=$ $1 / 2$ and $E_{c 4}=3 / 8$ (see Appendix E).

- The number of secondary paths $L$ in the simulated channel changes from 0 to 4 . The fading coefficient and delay for the paths are $\sigma_{0}=0.7, \tau_{0}=0$, $\sigma_{1}=0.6, \tau_{1}=5, \sigma_{2}=0.5, \tau_{2}=10, \sigma_{3}=0.4, \tau_{3}=15$, and $\sigma_{4}=0.3$, $\tau_{4}=20$.

- The spreading factor $\beta$ varies from 16 to 128 , the number of samples per chip $\lambda$ changes from 10 to 50, and the number of users $K$ varies from 1 to 10.

- The parameters used for computing the PMF of $C$ are $M=10^{6}, N=10^{3}$ and $C_{\max }=180$.

Fig. 4 shows the PMF graph of the variable $D$ computed according to Subsection 4.2 in the case of $\beta=64, \lambda=30, E_{b} / N_{0}=10 \mathrm{~dB}$ along with a gradual increment of $L$. We can see that when $L$ increases from 1 to 4 , the PMF changes. More specifically, the value region of $C_{p}^{*}$ is expanded in the increasing direction, while the value region of $P_{p}$ is reduced. This change is reflected in Eq. (40) where the BER is reduced with the increment of $L$. 


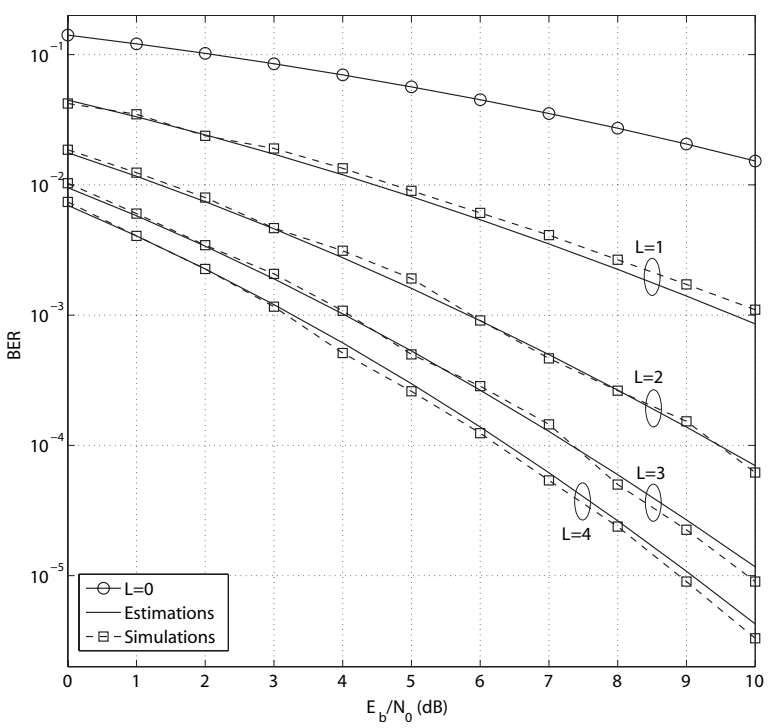

Fig. 5 BER performance in the case of $K=1, \beta=64, \lambda=30$ with the increment of $L$

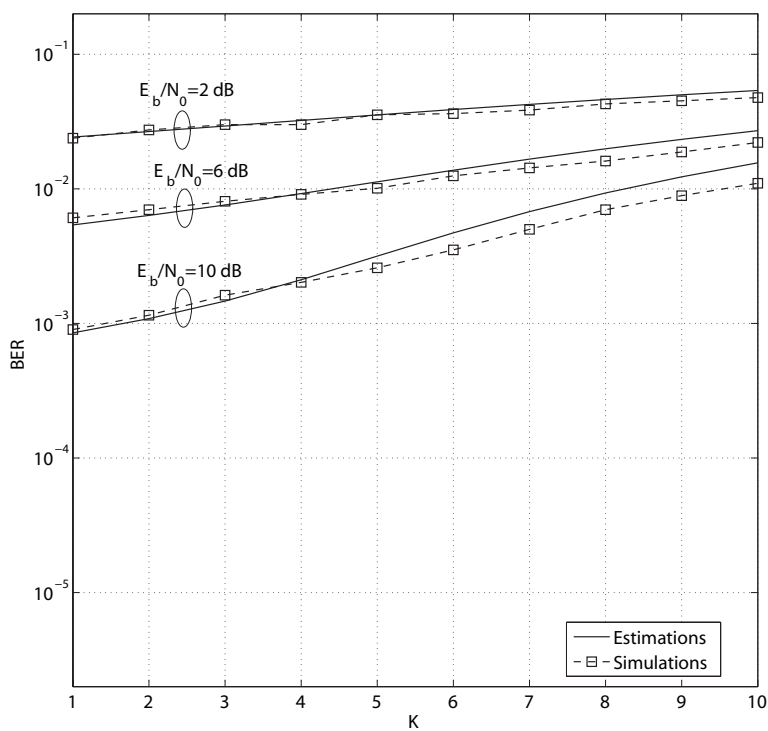

Fig. 6 BER values versus the number of users $K$ in the case of $L=1, \beta=64, \lambda=30$ with the increment of $E_{b} / N_{0}$

The estimated performance of a single-user system obtained by Eq. (40), for the cases of $L=1,2,3,4$, and Eq. (35) for $L=0$, are presented in Fig. 5, together with the corresponding simulated results. It can be observed that the simulation and the estimation results are nearly the same, while the system performance is improved significantly when the number of primary paths in- 


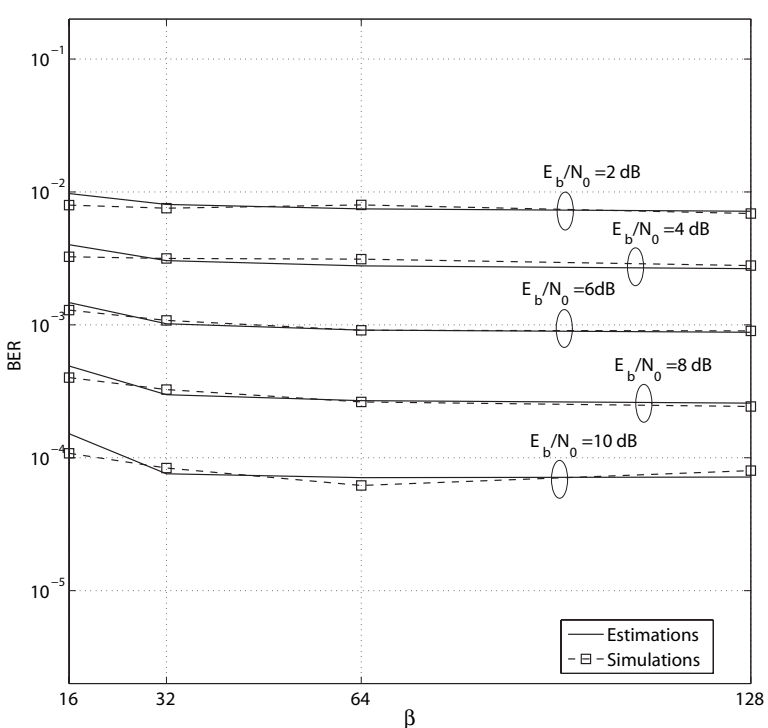

Fig. 7 BER values versus the spreading factor $\beta$ in the case of $K=1, L=2, \lambda=30$ with the increment of $E_{b} / N_{0}$

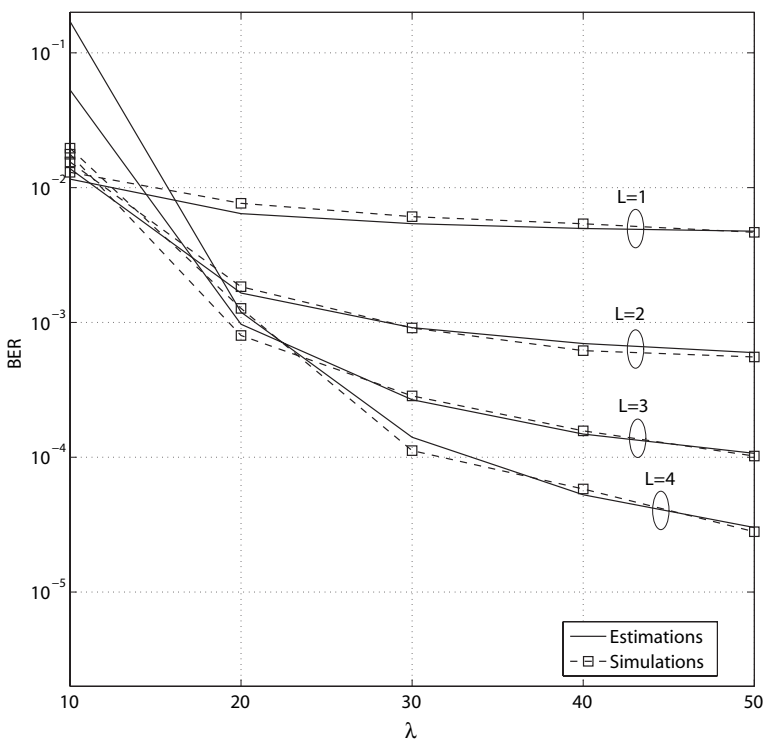

Fig. 8 BER values versus the number of samples per chip $\lambda$ in the case of $K=1, \beta=64$, $E_{b} / N_{0}=6 d B$ with the increment of $L$ 
creases. For example, at the same $E_{b} / N_{0}=8 d B$, the BER values for the cases of $L=0,1,2,3$ and 4 are equal to $3.6 \cdot 10^{-2}, 2.7 \cdot 10^{-3}, 2.6 \cdot 10^{-4}, 3.9 \cdot 10^{-5}$, and $2.4 \cdot 10^{-5}$, respectively.

The variations of the BER values depending on the number of users $K$ and the spreading factor $\beta$ with different values of $E_{b} / N_{0}$ are shown in Fig. 6 and Fig. 7, respectively. The BER performance becomes significantly worse with the increment of $K$ and slightly better with the increment of $\beta$. Specifically, at the same value of $E_{b} / N_{0}=10 \mathrm{~dB}$, the BERs obtained from simulation increase from $9.1 \cdot 10^{-4}$ to $1.1 \cdot 10^{-2}$ corresponding to $K$ changing from 1 to 10 , and decrease from $1.1 \cdot 10^{-4}$ to $8.1 \cdot 10^{-5}$ with $\beta$ changing from 16 to 128 , respectively. In general, the simulation results agree with those obtained by our estimation. However, with $K=5$ or $\beta=16$, slight differences between them start to be visible. If we continue to increase $K \geq 5$ or decrease $\beta \leq 16$, these differences become more and more clear. The reason is that the variables $A, B$ in Eq. (29) are approximated by the fixed values $\bar{A}, \bar{B}$ in Eq. (40) in order to make the BER estimation simpler.

In our chaos-based DS-CDMA system with invariable delays, the number of samples per chip $\lambda$ can be changed by either keeping the chip duration fixed and changing the sampling clock in the receiver, or by keeping the sampling clock constant and changing the chip duration. In the first case, despite the sampling clock changing, the ratio, i.e., $\tau_{j} / \lambda$, does not change. Note in Eqs. (29)-(32) that the BER only depends on the ratio $\tau_{j} / \lambda$ and not on the specific values of $\tau_{j}$ or $\lambda$. Therefore, the BER does not change in this case. In the second case, the simultaneous change of the chip duration $T_{c}$ in both the transmitter and the receiver leads to a change in the ratio $\tau_{j} / \lambda$ and thus in the BER of the system. Fig. 8 displays the BER values versus the number of samples per chip $\lambda$ for this second case. The simulation results show that the chaos-based DS-CDMA system performs better when $\lambda$ is increased. For example in case of $L=4$, the BER values are $2 \cdot 10^{-2}, 1.3 \cdot 10^{-3}, 1.1 \cdot 10^{-4}$, $5.8 \cdot 10^{-5}$ and $2.3 \cdot 10^{-5}$ corresponding to $\lambda=10,20,30,40$ and 50 . It can be observed that the estimation results have a good match with the simulation ones except for the cases of $L=3,4$ with $\lambda=10$. The cause of this mismatch is that the simulation parameters chosen for these cases, i.e., $\tau_{3}, \tau_{4}>T_{c}=\lambda$, are not suitable for the channel condition in theory, i.e., all delays $\leq T_{c}$.

\section{Conclusions}

In this paper we investigated for the first time the BER performance of directoversampling correlator-type receivers in chaos-based DS-CDMA systems over frequency non-selective fading channels. The mathematical models for the transmitting side, the generalized channel, and the proposed receiver are provided and described in detail. The system performance is approximately estimated by using theoretical derivation in combination with discrete numerical integral. Our results show that the proposed receiver has the following noticeable features: (1) It can perform well with flat fading channels, especially 
the BER performance is significantly enhanced when the number of secondary paths increases; (2) Due to its simple structure, the receiver is a promising alternative to RAKE receivers for the design of a secure physical layer in WSNs or LR-WPANs; (3) Since the receiver can perform the demodulation process based on discrete-sample processing, it could be easily implemented on programmable integrated circuits (IC) such as FPGA or DSP; (4) The use of an oversampling block at the input requires that all remaining blocks in the receiver work at higher clock frequencies. Although this increases the power consumption and cost of the solution, with the current development of the IC technology, we can easily implement the receiver with high speed, low consumption and low cost ICs. The hardware implementation of the proposed receiver is part of our future work.

\section{Appendix}

A. The expression for the output signal of the correlator:

$$
\begin{aligned}
s_{l}^{(i)} & =\sum_{g=1}^{\lambda \beta} d_{g, k, l}^{(i)}=\sum_{k=1}^{\beta} \sum_{g=1}^{\lambda} d_{g, k, l}^{(i)}=\sum_{k=1}^{\beta} \sum_{g=1}^{\lambda} r_{g, k, l} x_{k, l}^{(i)} \\
& =\sum_{k=1}^{\beta}\left(\lambda \alpha_{0} x_{k, l}^{(i)} \sum_{i=1}^{K} b_{l}^{(i)} x_{k, l}^{(i)}+\left(\lambda-\tau_{1}\right) \alpha_{1} x_{k, l}^{(i)} \sum_{i=1}^{K} b_{l}^{(i)} x_{k, l}^{(i)}+\ldots+\left(\lambda-\tau_{L}\right) \alpha_{L} x_{k, l}^{(i)} \sum_{i=1}^{K} b_{l}^{(i)} x_{k, l}^{(i)}\right. \\
& \left.+\tau_{1} \alpha_{1} x_{k, l}^{(i)} \sum_{i=1}^{K} b_{l}^{(i)} x_{k-1, l}^{(i)}+\ldots+\tau_{L} \alpha_{L} x_{k, l}^{(i)} \sum_{i=1}^{K} b_{l}^{(i)} x_{k-1, l}^{(i)}+\lambda \eta_{k, l} x_{k, l}^{(i)}\right) \\
& =\left(\sum_{j=0}^{L} \lambda \alpha_{j}\right)\left(\sum_{k=1}^{\beta} b_{l}^{(i)} x_{k, l}^{(i)}+\sum_{k=1}^{\beta} \sum_{m=1, m \neq i}^{K} b_{l}^{(m)} x_{k, l}^{(m)} x_{k, l}^{(i)}\right)+\left(\sum_{j=1}^{L} \tau_{j} \alpha_{j}\right)\left(-\sum_{k=1}^{\beta} b_{l}^{(i)} x_{k, l}^{(i)}{ }^{2}\right. \\
& \left.-\sum_{k=1}^{\beta} \sum_{m=1, m \neq i}^{K} b_{l}^{(m)} x_{k, l}^{(m)} x_{k, l}^{(i)}+\sum_{k=1}^{\beta} \sum_{m=1, m \neq i}^{\beta} b_{l}^{(m)} x_{k-1, l}^{(m)} x_{k, l}^{(i)}\right)+\lambda \sum_{k=1} \eta_{k, l} x_{k, l}^{(i)}
\end{aligned}
$$

B. The statistical calculation for the mean values of $X, Y$ :

$$
\begin{aligned}
E\left[X \mid b_{l}^{(i)}=+1, \alpha_{j}\right] & =\left(\sum_{j=0}^{L} \lambda \alpha_{j}\right)\left(E\left[\sum_{k=1}^{\beta} x_{k, l}^{(i)}{ }^{2}\right]+E\left[\sum_{k=1}^{\beta} \sum_{m=1, m \neq i}^{K} b_{l}^{(m)} x_{k, l}^{(m)} x_{k, l}^{(i)}\right]\right) \\
& =\left(\sum_{j=0}^{L} \lambda \alpha_{j}\right) \sum_{k=1}^{\beta} E\left[x_{k, l}^{(i)^{2}}\right]=\left(\sum_{j=0}^{L} \lambda \alpha_{j}\right) \beta E_{c},
\end{aligned}
$$




$$
\begin{aligned}
E\left[Y \mid b_{l}^{(i)}=+1, \alpha_{j}\right] & =\left(\sum_{j=1}^{L} \tau_{j} \alpha_{j}\right)\left(-E\left[\sum_{k=1}^{\beta} x_{k, l}^{(i)}{ }^{2}\right]-E\left[\sum_{k=1}^{\beta} \sum_{m=1, m \neq i}^{K} b_{l}^{(m)} x_{k, l}^{(m)} x_{k, l}^{(i)}\right]\right. \\
& \left.+E\left[\sum_{k=1}^{\beta} \sum_{m=1, m \neq i}^{K} b_{l}^{(m)} x_{k-1, l}^{(m)} x_{k, l}^{(i)}\right]\right) \\
& =\left(\sum_{j=1}^{L} \tau_{j} \alpha_{j}\right)\left(-\sum_{k=1}^{\beta} E\left[x_{k, l}^{(i)}{ }^{2}\right]\right)=-\left(\sum_{j=1}^{L} \tau_{j} \alpha_{j}\right) \beta E_{c},
\end{aligned}
$$

C. The statistical calculation for the mean squared values of $X, Y, Z$ :

$$
\begin{aligned}
E\left[X^{2} \mid b_{l}^{(i)}=+1, \alpha_{j}\right] & =\left(\sum_{j=0}^{L} \lambda \alpha_{j}\right)^{2}\left(E\left[\left(\sum_{k=1}^{\beta} x_{k, l}^{(i)}\right)^{2}\right)^{2}+2 E\left[\sum_{k=1}^{\beta} x_{k, l}^{(i)} \sum_{k=1}^{\beta} \sum_{m=1, m \neq i}^{K} b_{l}^{(m)} x_{k, l}^{(m)} x_{k, l}^{(i)}\right]\right. \\
& \left.+E\left[\left(\sum_{k=1}^{\beta} \sum_{m=1, m \neq i}^{K} b_{l}^{(m)} x_{k, l}^{(m)} x_{k, l}^{(i)}\right)^{2}\right]\right) \\
& =\left(\sum_{j=0}^{L} \lambda \alpha_{j}\right)^{2}\left(\sum_{k=1}^{\beta} E\left[x_{k, l}^{(i)^{4}}\right]+\sum_{k=1}^{\beta-1} E\left[x_{k, l}^{(i)^{2}}\right] \sum_{p=1, p \neq k}^{\beta} E\left[x_{p, l}^{(i)}\right]\right. \\
& \left.+\sum_{k=1}^{\beta} E\left[x_{k, l}^{(i)}\right]^{2} \sum_{m=1, m \neq i}^{K} E\left[x_{k, l}^{(m)^{2}}\right]\right) \\
& =\left(\sum_{j=0}^{L} \lambda \alpha_{j}\right)^{2}\left(\beta E_{c 4}+\beta(\beta-1) E_{c}^{2}+\beta(K-1) E_{c}^{2}\right) \\
& =\left(\sum_{j=0}^{L} \lambda \alpha_{j}\right)^{2}\left(\beta E_{c 4}+\beta(\beta+K-2) E_{c}^{2}\right) .
\end{aligned}
$$




$$
\begin{aligned}
E\left[Y^{2} \mid b_{l}^{(i)}=+1, \alpha_{j}\right] & =\left(\sum_{j=1}^{L} \tau_{j} \alpha_{j}\right)^{2}\left(E\left[\left(\sum_{k=1}^{\beta} x_{k, l}^{(i)}\right)^{2}\right]+E\left[\left(\sum_{k=1}^{\beta} \sum_{m=1, m \neq i}^{K} b_{l}^{(m)} x_{k, l}^{(m)} x_{k, l}^{(i)}\right)^{2}\right]\right. \\
& \left.+E\left[\left(\sum_{k=1}^{\beta} \sum_{m=1, m \neq i}^{K} b_{l}^{(m)} x_{k-1, l}^{(m)} x_{k, l}^{(i)}\right)^{2}\right]\right) \\
& =\left(\sum_{j=1}^{L} \tau_{j} \alpha_{j}\right)^{2}\left(\beta E_{c 4}+\beta(\beta-1) E_{c}^{2}+\beta(K-1) E_{c}^{2}+\beta K E_{c}^{2}\right) \\
& =\left(\sum_{j=1}^{L} \tau_{j} \alpha_{j}\right)^{2}\left(\beta E_{c 4}+\beta(\beta+2 K-2) E_{c}^{2}\right) .
\end{aligned}
$$

$E\left[Z^{2} \mid b_{l}^{(i)}=+1\right]=\lambda^{2} E\left[\left(\sum_{k=1}^{\beta} \eta_{k, l} x_{k, l}^{(i)}\right)^{2}\right]=\lambda^{2} \sum_{k=1}^{\beta} E\left[\eta_{k, l}^{2}\right] E\left[x_{k, l}^{(i)^{2}}\right]=\lambda^{2} E_{b} \frac{N_{0}}{2}$

D. The rough BER expression obtained by the theoretical derivation:

$$
\begin{aligned}
B E R_{R} & =\frac{1}{2} \operatorname{Prob}\left(s_{l}^{(i)} \leq 0 \mid b_{l}^{(i)}=+1, \alpha_{j}\right)+\frac{1}{2} \operatorname{Prob}\left(s_{l}^{(i)}>0 \mid b_{l}^{(i)}=-1, \alpha_{j}\right) \\
& =\operatorname{Prob}\left(s_{l}^{(i)} \leq 0 \mid b_{l}^{(i)}=+1, \alpha_{j}\right)=Q\left(\frac{\operatorname{Var}\left[s_{l}^{(i)} \mid b_{l}^{(i)}=+1, \alpha_{j}\right]}{E^{2}\left[s_{l}^{(i)} \mid b_{l}^{(i)}=+1, \alpha_{j}\right]}\right)^{-\frac{1}{2}} \\
& \left.=Q\left(\frac{\left.\left(\sum_{j=0}^{L} \lambda \alpha_{j}\right)^{2}\left(\beta E_{c 4}+\beta(K-2) E_{c}^{2}\right)+\left(\sum_{j=1}^{L} \tau_{j} \alpha_{j}\right)^{2}\left(\beta E_{c 4}+\beta(2 K-2) E_{c}^{2}\right)+\lambda^{2} \beta \frac{N_{0}}{2} E_{c}\right)^{-\frac{1}{2}}}{\left(\sum_{j=0}^{L} \lambda \alpha_{j}-\sum_{j=1}^{L} \tau_{j} \alpha_{j}\right)^{2} \beta^{2} E_{c}^{2}}\right)\right)^{-\frac{1}{2}} \\
& =Q\left(\frac{\frac{E_{c 4}}{E_{c}^{2}}+K-2}{\beta\left(1-\frac{E_{j=1}^{L}}{\sum_{j=0}^{L} \alpha_{j}}\right)^{2}}+\frac{\frac{E_{c 4}}{E_{c}^{2}}+2 K-2}{\beta\left(\frac{\sum_{j=0}^{L} \alpha_{j}}{\sum_{j=1}^{L} \frac{\tau_{j}}{\lambda} \alpha_{j}}-1\right)^{2}}+\frac{1}{2\left(\sum_{j=0}^{L} \alpha_{j}-\sum_{j=1}^{L} \frac{\tau_{j}}{\lambda} \alpha_{j}\right)^{\frac{E_{b}}{N_{0}}}}\right)^{2} \\
& =Q\left(A+B+\frac{1}{2 C}\right)^{-\frac{1}{2}} \cdot
\end{aligned}
$$


E. The statistical properties of the Chebyshev polynomial function of order 2 : The invariant PDF of $x$, denoted by $\rho(x)$ is

$$
\rho(x)= \begin{cases}\frac{1}{\pi \sqrt{1-x^{2}}} & |x|<1, \\ 0 & \text { otherwise. }\end{cases}
$$

The values of $E_{c}$ and $E_{c 4}$ are calculated as

$$
\begin{aligned}
& E_{c}=E\left[x_{k, l}^{(i)^{2}}\right]=\int_{-\infty}^{\infty} x^{2} \rho(x) d x=\int_{-1}^{1} x^{2} \frac{1}{\pi \sqrt{1-x^{2}}} d x=\frac{1}{2} . \\
& E_{c 4}=E\left[x_{k, l}^{(i)^{4}}\right]=\int_{-\infty}^{\infty} x^{4} \rho(x) d x=\int_{-1}^{1} x^{4} \frac{1}{\pi \sqrt{1-x^{2}}} d x=\frac{3}{8} .
\end{aligned}
$$

\section{Acknowledgment}

This research was carried out in framework of the AREAS+ scholarship program in Erasmus Mundus Action 2 project. It was also partially supported by the Spanish Ministry of Economy and Competitiveness and EU FEDER under grant TEC2014-59583-C2-2-R (SUNSET project) and by the Catalan Government (ref. 2014SGR-1427).

\section{References}

1. Andrew J. Viterbi, CDMA: Principles of Spread Spectrum Communication (AddisonWesley, 1 edition, 1995)

2. R. L. Peterson, R. E. Zeimer, and D. E. Borth, Introduction to Spread Spectrum Communications ( Prentice Hall, New York, NY, USA, 1995)

3. P. Stavroulakis, Chaos Applications in Telecommunications (CRC Press, 2005)

4. G. Heidari-Bateni and C. D. McGillem, Chaotic sequences for spread spectrum: an alternative to PN-sequences, in IEEE Int. Conf. Sel. Topics Wireless Communications, Vancouver, Canada, 1992, pp. 437-440

5. J. Yu and Y.-D. Yao, Detection performance of chaotic spreading LPI waveforms. IEEE Trans. Wireless Commun. 4, pp. 390-396 (2005)

6. G. Heidari-Bateni and C. D. McGillem, A chaotic direct-sequence spread-spectrum communication system. IEEE Transactions on Communications 42, pp. 1524-1527 (1994)

7. Q. Zhang and J. Zheng, Choice of chaotic spreading sequences for asynchronous DSCDMA communication, in Proc. IEEE Asia-Pacific Conf. CAS, 2000, pp. 642-645

8. L. Cong and L. Shaoquian, Chaotic spreading sequences with multiple access performance better than random sequence. IEEE Trans. Circuits. Syst. I 47, pp. 394-397 (2000)

9. G. Mazzini, G. Setti, and R. Rovatti, Chaotic complex spreading sequences for asynchronous DS-CDMA. I. System modeling and results. IEEE Tr.. Circ. Syst. I 44, pp. 937-947(1997)

10. R. Rovatti, G. Setti, and G. Mazzini, Chaotic complex spreading sequences for asynchronous DS-CDMA. Part II. Some theoretical performance bounds. IEEE Trans. Circuits Syst. I 45, pp. 496-506 (1998)

11. C. Chen, K. Yao, K. Umeno, E. Biglieri, Design of spread-spectrum sequences using chaotic dynamical systems and ergodic theory. IEEE Trans. Circuits Syst. 48, pp. 11101114 (2001) 
12. R. Rovatti, G. Setti, G. Mazzini, Toward sequence optimization for chaos-based asynchronous DS-CDMA systems, in Proc. IEEE GLOBECOM, Sydney, Australia, 1998, pp. 2174-2179

13. G. Setti, R. Rovatti, and G. Mazzini, Synchronization Mechanism and Optimization of Spreading Sequences in Chaos-Based DS-CDMA Systems. IEICE TRANS. on Fundamentals of Elec., Commun. and Computer Sciences E82-A, pp. 1737-1746 (1999)

14. B. Jovic, C. Unsworth, G. Sandhu, and S. Berber, A robust sequence synchronization unit for multi-user DS-CDMA chaos-based communication systems. Signal Processing 87, pp. 1692-1708 (2007)

15. G. Kaddoum, D. Roviras, P. Charge, and D. Fournier-Prunaret, Robust synchronization for asynchronous multi-user chaos-based DS-CDMA. Signal Processing 89, pp. 807-818 (2009)

16. R. Vali, S. Berber, and S. Nguang, Effect of Rayleigh fading on noncoherent sequence synchronization for multi-user chaos based DS-CDMA. Signal Processing 90, pp. 19241939 (2010)

17. S. Azou, C. Pistre, L. L. Duff, and G. Burel, Sea trial results of a chaotic direct sequence spread spectrum underwater communication system. in Proc. IEEE Oceans, San Diego, Calif, USA, 2003, pp. 1539-1546

18. W. M. Tam, F. C. M. Lau, C. K. Tse, and M. M. Yip, An approach to calculating the bit-error rate of a coherent chaos shift-keying digital communication system under a noisy multiuser environment. IEEE Transactions on Circuits and Systems I 49, pp. 210-223 (2002)

19. S. Vitali, R. Rovatti, and G. Setti, On the performance of chaos-based multicode DSCDMA systems. Circuits, Systems, and Signal Processing 24, pp. 475-495 (2005)

20. A. J. Lawrance and G. Ohama, Exact calculation of bit error rates in communication systems with chaotic modulation. IEEE Trans. on Circuits and Systems I 50, pp. 1391-1400 (2003)

21. W. Tam, F. Lau, C. Tse, and A. Lawrance, Exact analytical bit error rates for multiple access chaos-based communication systems. IEEE Trans. Circuits Syst. II 51, pp. 473-481 (2004)

22. G. Kaddoum, P. Charge, D. Roviras, D. Fournier-Prunaret, A Methodology for Bit Error Rate Prediction in Chaos-based Communication Systems. Circuits Syst Signal Processing 28, pp. 925-944 (2009)

23. G. Kaddoum, P. Charge, and D. Roviras, A Generalized Methodology for Bit-ErrorRate Prediction in Correlation-Based Communication Schemes Using Chaos. IEEE Comm. Letters 13, pp. 567-569 (2009)

24. R. Rovatti, G. Mazzini, and G. Setti, Enhanced rake receivers for chaos-based DSCDMA. IEEE Trans. Circuits and Sys. I 48, pp. 818-829 (2001)

25. G. Mazzini, R. Rovatti, and G. Setti, Chaos-based asynchronous DS-CDMA systems and enhanced rake receivers: measuring the improvements. IEEE Transactions on Circuits and Systems I 48, pp. 1445-1453 (2001)

26. G. Kaddoum, D. Roviras, P. Charge, and D. Fournier-Prunaret, Accurate bit error rate calculation for asynchronous chaos-based DS-CDMA over multipath channel. EURASIP Journal on Advances in Signal Processing, vol. 2009, ID 571307, 12 pages (2009)

27. G. Kaddoum, M. Coulon, D.Roviras, and P. Charge, Theoretical performance for asynchronous multi-user chaos-based communication systems on fading channels. Elsevier Signal Processing 90, pp. 2923-2933 (2010)

28. E. N. Gilbert, Increased Information Rate by Oversampling. IEEE Transactions on Information Theory 39, pp.1973-1976 (1993).

29. B. El-Khaldi, J. M. Rouvaen, A. Menhaj, Y. El hillali, Averaging and oversampling correlator receiver with input quantization. Digital Signal Processing 16, pp. 120136 (2006).

30. Y. S. Lee and B. S. Seo, OFDM receivers using oversampling with rational sampling ratios. IEEE Trans. Consumer Electronics 55, pp. 1765-1770 (2009).

31. S. Berber and N. Chen, Physical Layer Design in Wireless Sensor Networks for Fading Mitigation. J. Sens. Actuator Netw. 2, pp. 614-630 (2013)

32. IEEE Standard for Local and Metropolitan Area Networks-Part 15.4: Low-Rate Wireless Personal Area Networks (LR-WPANs). IEEE Standard 802.15.4-2011(Revision of IEEE Std 802.15.4-2006); IEEE: New York, NY, USA, 2011; pp. 1-294 
33. A. F. Molisch, K. Balakrishnan, D. Cassioli, C.-C. Chong, S. Emami, A. Fort, J. Karedal, J. Kunisch, H. Schantz, U. Schuster, and K. Siwiak, Ieee 802.15.4a channel model final report, The Institution of Electrical Engineers.

34. M. K. Simon and MS. Alouini, Fading Channel Characterization and Modeling, in Digital Communication over Fading Channels-A Unified Approach to Performance Analysis, CRC Press, 2005

35. C.C. Wang and et. al, Zig-Bee 868/915-MHz modulator/demodulator for wireless personal area network. IEEE Trans. Very-Large Scale Integr.(VLSI) Syst. 16, pp. 936-938 (2008)

36. N. J. Oh, S. G. Lee, Building a 2.4-GHZ radio transceiver using IEEE 802.15.4. IEEE Circuits Devices Mag. 21, pp. 43-51 (2006)

37. M. A. Al-Jarraha, N. K. Al-Ababnehb, M. M. Al-Ibrahimb, R. A. Al-Jarrah, Cooperative OFDM for semi distributed detection in wireless sensor networks. AEU Int. Jour. of Elect. and Commu. 68, pp. 1022-1029 (2012)

38. M. R. Islam, Y. S. Han, Cooperative MIMO communication at wireless sensor network: An error correcting code approach. J. Sens. Actuator Netw. 11, pp. 9887-9903 (2011)

39. S. M. Berber, Probability of error derivatives for binary and chaos-based CDMA systems in wide-band channels. IEEE Trans. Wireless Comm. 13, pp. 5596-5606 (2014)

40. M. M. Siddiqui, Statistical inference for Rayleigh distributions. Journal of Research of the National Bureau of Standards Vol. 68D, pp. 1007 (1964)

41. C. M. Grinstead and J. L. Snell, Central Limit Theorem, Introduction to Probability (American Mathematical Society, 2nd Revised edition, July 1997), pp. 325-364. 